\title{
UNEQUAL ITERATIVE DECODING FOR POWER EFFICIENT VIDEO TRANSMISSION
}

\author{
Yongfang Wang, Songyu Yu, Xiaokang Yang \\ Institute of Image Communication and Information Processing, \\ Shanghai Jiao Tong University, Shanghai 200240, China
}

\begin{abstract}
We present an unequal iterative decoding (UID) approach for minimization of the receiver power consumption subject to a given quality of service, by exploiting data partitioning and turbo decoding. We assign unequal decoding iterations of forward error correction (FEC) to data partitions with different importance by jointly considering the source coding, channel coding and receiver power allocation. The proposed scheme has been applied to H.264 video over AWGN channel, and achieves excellent tradeoff between video delivery quality and power consumption, and yields significant power saving compared with the classical equal iterative decoding (EID) approach in wireless video transmission.
\end{abstract}

\section{INTRODUCTION}

With the wireless network explosion, robust transmission of compressed video over wireless channel has become an increasingly important requirement for wireless multimedia systems. To improve error resilience in error-prone environment, the prevalent video coding standards, MPEG4 [1] and H.264 [2], adopts data partitioning tools, which partition the data in a coding unit into several parts with different importance. Unequal error protection methods combing forward error correction (FEC) and data partitioning [3]-[4] are promising solutions for wireless video application. These algorithms apply FEC codes of varying rates to a number of data fragments according to the importance of the data at encoder. However, they only considered optimal bit-allocation schemes for joint source channel coding, but did not consider power consumption or battery life constraint. [5]-[7] consider the processing power for source coding and channel coding as well as transmission power for a given video quality, but only RS code was considered.

In this paper, we propose an unequal iterative decoding (UID) approach by exploiting data partitioning and turbo decoding. It is motivated by the fact that as the number of iterations used by the turbo decoder increases, the turbo decoder performs significantly better. We give the data with high priority more number of iterations used by the turbo decoder. The advantage of this method is that different degrees of protection are achieved at given channel rates and processed only at decoder. Simulation results in H.264 demonstrate that this proposed algorithm not only achieves significant tradeoff between video delivery quality and power consumption but also reduces power consumption compared with classical equal iterative decoding (EID) approach. The proposed simple yet efficient UID approach provides an additional power saving option to mobile video applications, especially in portable application, such as mobile telephone phones and wireless multimedia terminals.

\section{UNEQUAL ITERATIVE DECODING FOR TURBO CODE PROTECTED VIDEO TRANSMISSION}

\subsection{System Description}

The proposed video transmission system is shown in Fig.1. In this system, firstly, the original video is compressed into different important partitions/streams by video encoder, which are generated according to the different importance for the quality of the reconstructed video. Secondly, these compressed streams are encoded by turbo encoders at the same rates. Besides, the channel-coded streams are modulated and then are transmitted over wireless channel. At the receiver, the UID scheme is employed to provide different levels of turbo decoding to different streams. We expect to improve the video quality with the turbo successive iterations. Lastly, we reconstruct video stream by video decoder with error concealment.

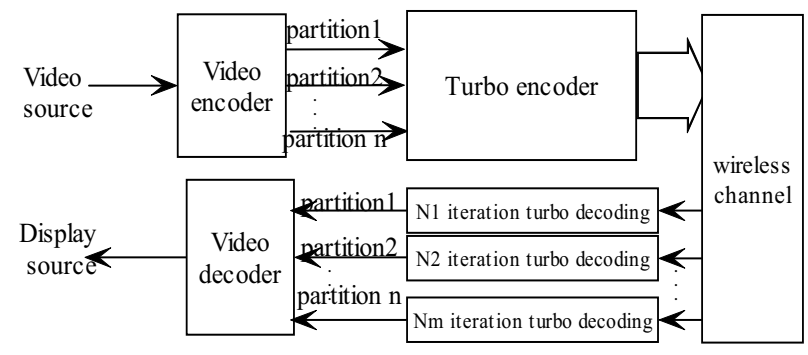

Fig.1 System architecture of video transmission based UID

\subsection{Tradeoff between Coding Efficiency and Power}


To design an efficient UID scheme, we consider video quality and power consumption jointly. In this paper, we fix this rate of channel codes and rely on the optimal iteration assignment of turbo decoding to handle channel variations thanks to the operational distortion-rate function $D(R)$ of the video coder and the power consumption function $P(R)$ of decoding.

We define the rate-distortion function as

$$
D(R)=\sum_{i=0}^{n-1}\left(D_{s}\left(R_{s}^{i}\right)+D_{c}\left(R_{c}^{i}, R_{s}^{i}, P_{e}^{i}\right)\right)
$$

where $n$ indicates the number of video stream partitioning. $R_{s}^{i}$ means the source bit-rate for ith partition. $R_{c}^{i}$ is the channel redundancy bits for $i t h$ partition. $P_{e}^{i}$ denotes the equivalent channel bit error rate after channel decoding for the ith partition. $D_{s}\left(R_{s}^{i}\right)$ means the source distortion for ith partition. $D_{c}\left(R_{c}^{i}, R_{s}^{i}, P_{e}^{i}\right)$ is the channel distortion for ith partition. The overall bit-rate can be written as

$$
R=\sum_{i=0}^{n-1}\left(R_{s}^{i}+R_{c}^{i}\right)
$$

where $R$ indicates the total bit-rate.

In [11], a more exact estimation of the bit error rate as a function of the number of iterations can be for example be obtained via an EXIT chart. For simplicity, we find that the channel bit error rate is monotonically decreasing with increasing iterative number of turbo code. Therefore, it is reasonable that the channel bit error rate function can be approximated by

$$
P_{e}=\frac{\alpha}{e^{\beta N}}
$$

where $N$ is the number of decoding iteration. $\alpha$ and $\beta$ are parameters to be determined, which are affected by channel rate, signal-to-noise (SNR) and so on. Using the nonlinear parameter estimation method, we can get the parameters $\alpha$ and $\beta$. Assuming independent bit errors, the packet loss probability for the $k t h$ packet can be calculated by

$\rho^{k}=1-\left(1-P_{e}\right)^{B_{k}}$

where $B_{k}$ is the packet size in bits. Note that the probability of packet loss $\rho^{k}$ is a function of turbo iterative number, source coding parameter, and the channel coding rate selected for the packet.

The total expected distortion function for the $k t h$ packet is given by [7]

$$
E\left[D^{k}\right]=\left(1-\rho^{k}\right) E\left(D_{R}^{k}\right)+\rho^{k} E\left(D_{L}^{k}\right)
$$

where $E\left(D_{R}^{k}\right)$ and $E\left(D_{L}^{k}\right)$ are the expected distortion for the $k$ th packet when the packet is either received correctly or lost, respectively, and $\rho^{k}$ is the loss probability of the $k t h$ source packet. The expected distortion if a packet is received, $E\left(D_{R}^{k}\right)$, depends only on the source-coding parameters for the packet. The expected distortion $E\left(D_{L}^{k}\right)$, if a packet is lost, depends on the concealment strategy used at the decoder.

$$
\begin{aligned}
& \text { So (1) can be rewritten as } \\
& \begin{aligned}
D(R) & =\sum_{i=0}^{n-1} \sum_{k=1}^{K_{i}} D\left(R_{s}^{i k}, R_{c}^{i k}, N_{i k}\right) \\
& =\sum_{i=0}^{n-1} \sum_{k=1}^{K_{i}}\left(\left(1-\rho^{i k}\right) E\left(D_{R}^{i k}\right)+\rho^{i k} E\left(D_{L}^{i k}\right)\right)
\end{aligned}
\end{aligned}
$$

where $K_{i}$ means the number of packet of the ith partition. $N_{i k}$ is the turbo number of iterations (NOI) for the $k t h$ packet of the ith partition, $E\left(D_{R}^{i k}\right)$ and $E\left(D_{L}^{i k}\right)$ are the expected distortion for the kth packet of the ith partition when the packet is either received correctly or lost, respectively, and $\rho^{i k}$ is the loss probability of the kth source packet of the ith partition.

In this paper, we only consider the power consumed at receiver, which consists of source decoding power and channel decoding power, which can be described as

$P(R)=P_{d s}\left(R_{s}\right)+P_{d c}\left(R_{s}, R_{c}, N\right)$

where $P_{d s}\left(R_{s}\right)$ is the power consumed by source decoding, $P_{d c}\left(R_{s}, R_{c}, N\right)$ is the power consumed by channel decoding. The source decoding power mainly consists of motion compensation, IDCT and quantization. The majority of the power consumption by the turbo decoding is the turbo number of iterations (NOI). The power consumption is proportional to the NOI. Thus as NOI increases, power consumption also increase. In [8], the number of operations per bit is approximately linear with the number of NOI. So (7) can also be written as

$$
\begin{aligned}
P(R) & =\sum_{i=0}^{n-1} r_{s} R_{s}^{i}+\sum_{i=0}^{n-1} r_{c}\left(R_{s}^{i}+R_{c}^{i}\right) \\
& =\sum_{i=0}^{n-1} r_{s} R_{s}^{i}+\sum_{i=0}^{n-1}\left(N_{i} \gamma+\lambda\right)\left(R_{s}^{i}+R_{c}^{i}\right)
\end{aligned}
$$

where $r_{s}$ is power consumed for per bit source decoding, $r_{c}$ is the power consumed for per bit channel decoding, $N_{i}$ is the NOI for the ith partition, the parameters $\gamma$ and $\lambda$ depend on the turbo decoding algorithm, channel SNR, and channel model.

It is observed that both the source side and the channel side have a tradeoff between the coding performance and power consumption. Thus, our problem can be formulated 
as: given the fixed bandwidth capacity how to adjust the iterative number of turbo decoding to minimize the power dissipation while keeping the end-to-end distortion of the source constant. Under the desired end-to-end distortion range, let $R_{T}$ represent the total available bits, $D_{T}$ represent the tolerable distortion, then the power-minimized resource allocation can be described as

$$
\min P(R)=\min \left(\sum_{i=0}^{n-1} r_{s} R_{s}^{i}+\sum_{i=0}^{n-1} N_{i} \gamma\left(R_{s}^{i}+R_{c}^{i}\right)\right)
$$

Subject to

$$
\begin{aligned}
& D(R) \leq D_{T} \\
& R \leq R_{T} \\
& N_{i} \geq N_{i+m}(m=0,1, \ldots n-i)
\end{aligned}
$$

The above constraints (10) (11) and (12) are referred to as "end-end distortion", "bandwidth" and "descending priority" constraints. The power-minimized resource allocation can be solved subjective to the above-mentioned constraint (10) (11) and (12) using an optimization method. In the paper, we represent an optimal solution for problem (9). We use Lagarange multiplier methods to relax end to end distortion and bandwidth constraints and be used to convert the constrained problem (9) into the following unconstrained problem

$$
\min _{\left\{R_{s}, R_{c}, N_{i}\right\}} J_{\text {tot }}=P(R)+\lambda_{1}\left(D(R)-D_{T}\right)+\lambda_{2}\left(R-R_{T}\right)
$$

where $\lambda_{1}$ and $\lambda_{2}$ are the Lagrange multipliers. By appropriately choosing $\lambda_{1}$ and $\lambda_{2}$, the solution to (9) can be obtained within a convex-hull approximation [9] by solving (13) under "descending priority" constraints.

\section{PERFORMANCE ANALYSIS AND SIMULATION}

In this section, we present some simulation results for transmitting H.264 video over AWGN channel. At a source side, we use the reference software JM8.6 of H.264 [10] as our source encoder. The Foreman sequence with 100 frames at QCIF resolution is encoded with "IPPP...." structure. Frame rate is $30 \mathrm{fbs}$, and source-coding rate is $100 \mathrm{kbps}$. Each frame is used as one slice and partitioned into three streams as [2]. At a channel side, the turbo encoder consisted of two identical parallel concatenated recursive systematic convolutional (RSC) encoders with the information bits interleaved between the two encoders, with constraint length $\mathrm{k}=4$ and polynomial generators $(15,13)$. The block size used was 4550 bits. The decoding was performed using the Log-MAP algorithm [12].

To demonstrate the effectiveness of our scheme, we investigated the impact of channel errors to overall quality of H.264 reconstructed video at a wide range of bit error rate. As partitioning $\mathrm{A}$ is more important, assigning an appropriate protection level for partitioning A would maximize reconstructed video quality. We here use the time consumption to measure the power consumption of decoding algorithm.

1) UID Scheme: our joint source channel power control with unequal iterative decoding for different important stream.

2) EID Scheme: an equal iterative decoding with fixed source complexity and Eb/N0.

Performance of the above two schemes are compared under media quality requirements.

Fig. 2 shows the comparison results of the decoding time consumption using UID scheme and EID scheme. The measured average Y-PSNR is plotted as a function of decoding time consumption with different available Eb/N0 of $0.4 \mathrm{db}, 0.6 \mathrm{db}, 0.8 \mathrm{db}$, respectively. We can see that our proposed scheme saves more power compared with EID scheme at the same PSNR requirement. More specifically, power save gains for UID scheme is much in evidence when the bit error rate is higher. This is due to the flexible of UID scheme by varying the turbo decoding iterations in response to the different important streams to combat the channel error. In addition, the lower of the $\mathrm{Eb} / \mathrm{N} 0$, the more the total decoding power consumes, moreover, the more efficient our scheme compares with the EID scheme. From Fig.2 (a), at a PSNR value of 28, the proposed UID scheme outperforms the EID scheme more than $13 \%$ power gain.

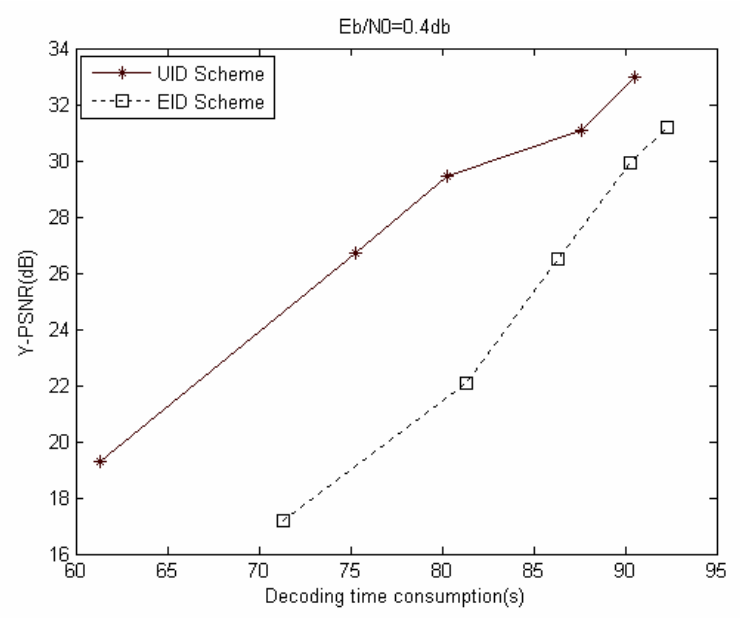

(a) 


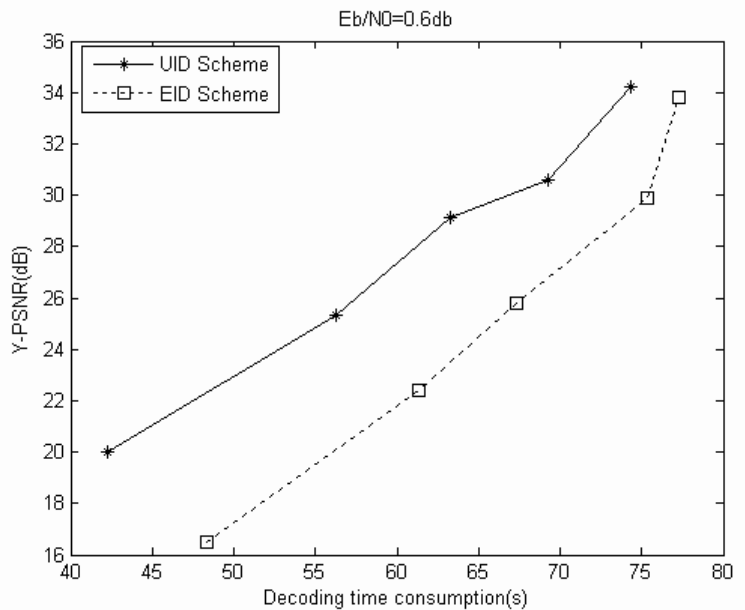

(b)

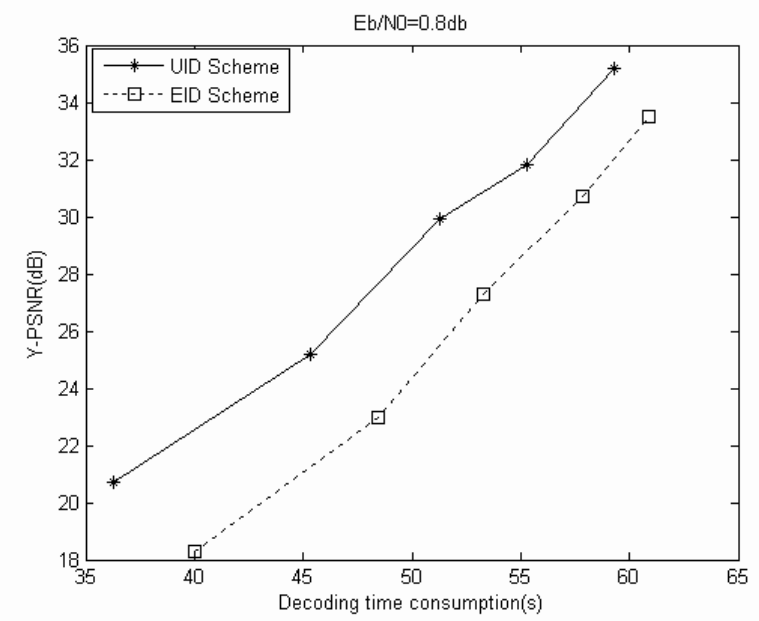

(c)

Fig.2 Comparisons of the power consumptions of UID scheme and EID scheme versus different PSNR requirement when the available $\mathrm{Eb} / \mathrm{N} 0$ is (a) $0.4 \mathrm{db}$ (b) $0.6 \mathrm{db}$ (C) $0.8 \mathrm{db}$

\section{CONCLUSION}

A method for efficiently utilizing decoding power in wireless video communications has been presented. The objective was to minimize the receiver power while meeting distortion constraints specified by the video application. Simulation results show that our proposed UID algorithm not only improves transmission performance but also reduces power consumption in real time communication application.
[1] ISO/IEC JTC1/SC 29/WG 11/14496, "Information technology coding of audio-visual objects (MPEG-4)-part 2: Visual," MPEG2001/N4350, 2001.

[2] Draft ITU-T recommendation and final draft international standard of joint video specification (ITU-T Rec.H.264/ISO/IEC 14496-10 AVC) Joint Video Team (JVT) of ISO/IEC MPEG and ITU-T VCEG, Mar.2003, Doc. JVT-G050.

[3] G.Cheung and A.Zakhor, "Bit allocation for joint source channel coding of scalable video," IEEE Trans. Image Processing, vol.9, pp. 340-356, Mar. 2000.

[4] A.Mohr,E.Ridkin, and R.Ladner, "Unequal Loss Protection: Graceful degradation of image quality over packet erasure channel through for-ward error correction," IEEE J. Select. Areas commu., vol.18, pp.819-828, June 2000 .

[5] X.Lu,E.Erkip,and Y.wang, "Power efficient multimedia communication over wireless channels," IEEE J. Select. Areas Commun., vol.21, pp.1738-1751, Dec 2003.

[6] Q.Zhang,Z.Ji,W,Zhu,and Y.Q.Zhang, "Power-minimized bit allocation for video communication over wireless channels," IEEE Trans Circuits Syst. Video Technol., vol. 6, pp. 398-410, June 2002.

[7]Y.Eisenberg,C.E.Luna,T.N.Pappas,R.Berry,andA.K.Katsaggels, "Joint source coding and transmission power management for energy efficient wireless video communications," IEEE Trans. On Circuits System Video Technology, vol.2, no.6, pp.411-424, June 2002

[8] L. T. Smit, G. J. Smit, P. J. Havinga, J. L. Hurink, and H. J. Broersma. "Influences of rake receiver/turbo decoder parameters on energy consumption and quality," In Proc. of 2002, International Conference On Third Generation Wireless and Beyond, pp.227-235, 2002.

[9] A. Ortega and K. Ramchandran, "Rate-distortion methods for image and video compression," IEEE Signal Process Mag., vol.15, no. 6, pp. 23-50, Nov. 1998.

[10] JM9.6. ftp://standard.polycom.com/referencesoftware.

[11] S. ten Brink, "Convergence Behavior of Iteratively Decoded Parallel Concatenated Codes," IEEE Trans. Commun., vol.49, no.10, pp.1727-1737, Oct. 2001.

[12] P. Robertson, E. Villebrun, and P. Hoeher,"A comparison of optimal and sub-optimal map decoding algorithms operating in the log domain." In Proc. International Conference on Communications (ICC), pp.1009-1013, June 1995.

\section{REFERENCES}

